Glyceryl Monostearate : Probing the Self Assembly of a Lipid Amenable To Surface Modification for Hepatic Targeting

\title{
Marwah, Megha
}

2018-09-27

Marwah, M , Magarkar , A, Ray , D , Aswal , V , Bunker , A \& Nagarsenker , M 2018 , '

Glyceryl Monostearate : Probing the Self Assembly of a Lipid Amenable To Surface

Modification for Hepatic Targeting ' , Journal of Physical Chemistry C , vol. 122 , no. 38 , pp.

22160-22169 . https://doi.org/10.1021/acs.jpcc.8b05931

http://hdl.handle.net/10138/326466

https://doi.org/10.1021/acs.jpcc.8b05931

unspecified

acceptedVersion

Downloaded from Helda, University of Helsinki institutional repository.

This is an electronic reprint of the original article.

This reprint may differ from the original in pagination and typographic detail.

Please cite the original version. 
GLYCERYL MONOSTEARATE: PROBING THE SELF ASSEMBLY OF A LIPID AMENABLE TO SURFACE MODIFICATION FOR HEPATIC TARGETING

Megha Marwah ${ }^{1}$, Aniket Magarkar ${ }^{2}$, Debes Ray $^{3}$, Vinod Aswal $^{3}$, Alex Bunker $^{2 *}$, Mangal Nagarsenker $^{1}$

${ }^{1}$ Department of Pharmaceutics, Bombay College of Pharmacy, Kalina, Santacruz (East), Mumbai, India.

${ }^{2}$ Drug research program, Division of Pharmaceutical Biosciences, Faculty of Pharmacy, University of Helsinki, Helsinki, Finland.

${ }^{3}$ Solid State Physics Division, Bhabha Atomic Research Centre, Trombay, Mumbai, India. ABSTRACT

Glyceryl monostearate (GMS) is a single tailed lipidic monoglyceride commonly used as a non-toxic food additive. In this study, we have investigated GMS, specifically its selfassembling properties and subsequent application in drug delivery. Results from in silico modelling, corroborated by complementary small-angle neutron scattering (SANS), demonstrated vesicle formation; associated phase transitions were analysed using differential scanning calorimetry (DSC); dynamic light scattering (DLS) revealed particle size alterations that occurred in the transition region. Spherical morphology of unilamellar vesicles was visualized using transmission electron microscopy (TEM) imaging. Further, hydrophilic and hydrophobic drug loading in GMS vesicles and their amenability to surface modification for hepatic targeting have, in this study, been both predicted through molecular simulation study and demonstrated experimentally. The influence of hepatotropic ligands on the stability of drug loaded GMS vesicles vis-à-vis cholesterol has also been investigated; The resulting GMS based drug delivery vehicle, its properties enhanced through surface decoration, is envisaged to achieve targeted delivery of its payload to hepatocytes.

*Corresponding author email: alex.bunker2@gmail.com 


\section{INTRODUCTION:}

The development of nanoscale drug carriers, a field known as nanomedicine, has seen significant development since its inception in the mid-90s. So far, lipid vesicles, also known as liposomes, are the leading form of nanocarrier, e.g. the very first approved therapy based on this technology, the drug Doxil ${ }^{\circledR 1,2}$. Additionally, in a more general context, liposomes are vesicular systems that have been shown to be very useful models for biomembranes ${ }^{3}$.

Liposomes are spherically organized molecular self-assemblies of lipid bilayers enclosing an aqueous core. They are composed of amphiphilic lipids: molecules with a polar headgroup and nonpolar tails, i.e. they exhibit biphasic proclivity. In their applications as both nanocarriers and biomembrane models they have proven extremely versatile; the formulation can be altered to fine tune their properties. For example, for the case of Doxil, cholesterol and phospholipids with poly(ethylene glycol) (PEG) functionalized to their headgroups have been added, to optimize the membrane properties and create a protective polymer corona respectively; several metaphorical dials and switches exist to tune the properties of a liposome.

Phospholipids are, however, not the only molecules capable of forming vesicular structures: for example, Kunitake and Okahata reported bilayer formation by two chained synthetic didodecyldimethylammonium bromide molecules with a layer thickness of 30-50 A, opening new avenues in the domain of vesicular drug delivery ${ }^{4}$; bilayer formation is a physicochemical phenomenon, not limited to natural lipid molecules ${ }^{3}$. Furthermore, spontaneous vesicle formation of single-tailed surfactants, for example cationic cetyltrimethylammonium tosylate and anionic sodium dodecyl benzenesulfonate, was noted by Kaler et $a l^{5,6}$; bilayer formation for single chained amphiphiles capable of mimicking conformational mobility of two-chained amphiphiles was seen to occur. This is made possible by the introduction of a rigid segment, multiple/multivalent headgroups, long alkyl chains $\left(\mathrm{C}_{10}-\mathrm{C}_{20}\right)$ and branched chains, into single chained amphiphiles ${ }^{3,7}$. It is thus evident that the potential for fine tuning vesicular structures for drug delivery applications can be extended further by moving on from phospholipids to a broader set of amphiphilic molecules from which to form their metaphorical building blocks.

Monoglycerides are such single-tailed amphiphiles and possess the property of 'self-assembly' into various mesophasic, thermodynamically stable, liquid crystalline phases, e.g. lamellar crystalline, liquid crystalline, inverted bicontinuous, cubic or hexagonal phases. When dispersed in water, they form micelle, bilayer or vesicular morphologies, depending on 
conditions. Their formation, investigation and applications in drug delivery and food technology have been reviewed by Sagalowicz et $a l^{8}$.

Glyceryl monostearate (GMS) is a single-chained amphiphilic monostearyl ester of glycerol, with a long alkyl chain $\left(\mathrm{C}_{18}\right)$ and two oxygens in the head group. It holds significant importance in both the food and cosmetics industries, as emulsifier and emollient; GMS is easy to digest and non-toxic to cells and tissues. As such it has also been used in drug delivery, as both a selfnanoemulsifying drug delivery system ${ }^{9}$ and a solid lipid component of solid lipid nanoparticles for the delivery of lipophilic drugs, e.g. mefenamic acid ${ }^{10}$, paclitaxe ${ }^{11}$, dibenzoyl peroxide, erythromycin (base) and triamcinolone acetonide ${ }^{12}$. Other applications have also been reported, e.g. implants for gentamicin delivery ${ }^{13}$ and polymer-lipid nanoparticles for doxycycline hydrochloride delivery ${ }^{14}$. Mishra et al. have demonstrated $\mathrm{pH}$ dependent vesicle formation for stearic acid in presence of GMS with ciprofloxacin hydrochloride entrapped as a model drug ${ }^{15}$, thus mimicking two chained amphiphiles for bilayer formation.

The work described in this paper aims to further exploit the 'self-assembly' of GMS into unilamellar vesicles, in particular regarding possible applications in the delivery of both watersoluble and lipophilic drugs. We have investigated mechanistic aspects of vesicle formation through in vitro studies coupled with molecular dynamics (MD) simulations of the entrapment of water soluble and lipid soluble molecules by GMS vesicles; the in vitro techniques used include SANS, DLS, DSC and TEM. Furthermore, we studied the effect of surface modification of GMS vesicles with a hepatotropic ligand (targeting moiety) synthesized by us, as a test case; the influence of the targeting ligand on the physicochemical properties of GMS vesicles was also studied using our combined in vitro, in silico protocol.

This study represents the next step in our continuing effort ${ }^{2}$ to use computational molecular dynamics modelling, in synergistic combination with complementary experimental means of evaluation, as a tool to develop nanoscale vesicle based drug delivery systems. Until now we have only studied phospholipid based systems, liposomes; this study represents the next step of extending our protocol to other molecules capable of forming vesicles: we are proposing a new vesicle based drug delivery system based on GMS molecules and have combined computational and experimental methodologies to investigate their potential. 
2. Materials and Methods:

2.1 Materials:

GMS was obtained from Croda International Plc. Cholesterol was procured from SD Fine Chemicals, India. 5-fluorouracil (5-FU) was a kind gift from Piramal life sciences. Decoquinate (DQN) was procured from TCI chemicals. $\mathrm{D}_{2} \mathrm{O}(99 \%)$ was obtained from Sigma Aldrich. All solvents used were of analytical grade. Hepatospecific ligand molecules were synthesized in our laboratory.

2.2 Formulation of vesicles:

Vesicles were prepared by the conventional thin film hydration method using a solvent system consisting of chloroform and methanol. Briefly, 0.27 mmoles of GMS were dissolved in the solvent system to formulate plain GMS vesicles. Surface modified vesicles were prepared using a molecular mixture of GMS and hepatospecific ligand in chloroform and methanol. This was subjected to rotary evaporation under vacuum (Buchi) for solvent removal to yield a thin film of lipids which was hydrated with distilled water by heating above the phase transition temperature of lipids. The resultant dispersion was probe sonicated (Branson Sonifier 250, Danbury, USA) at $40 \%$ amplitude using a $2 \mathrm{sec}$ on, $1 \mathrm{sec}$ off pulse cycle for two minutes to get the desired size.

2.3 Characterization of vesicles: Confirmatory studies:

2.3.1 Determination of trapped volume using a water-soluble drug, 5-FU:

5-FU, a water-soluble molecule, was incorporated in the aqueous phase of plain GMS vesicles using the thin film hydration method, to determine the trapped volume of GMS vesicles. Sephadex G-50 loaded column was employed to separate free 5-FU from the entrapped 5-FU. Briefly, $200 \mu \mathrm{L}$ of the formulation was gently loaded on top of the column and eluted with distilled water to achieve separation of 5-FU. Quantification of 5-FU in the fractions collected upon elution was carried out spectrophotometrically at $\lambda$ max of $267 \mathrm{~nm}$ using a UV-visible spectrophotometer (Jasco V-530). Trapped volume of the vesicles was determined in duplicate based on amount of drug entrapped in vesicles and moles of lipid employed.

2.3.2 Determination of entrapment efficiency of drug loaded GMS vesicles

Apart from water soluble 5-FU, DQN, a hydrophobic drug, was separately incorporated into the vesicular system by solubilizing it in organic solvents along with GMS at a molar ratio of 
1:117 of DQN: GMS. Entrapment efficiency of 5-FU and DQN in GMS vesicles was determined using the ultracentrifugation method in duplicate. The formulation was subjected to ultracentrifugation (Beckman Coulter Optima Max XP) at 80,000 rpm for 1 hour at $4{ }^{\circ} \mathrm{C}$ resulting in separation of the free drug in supernatant from that entrapped within vesicles obtained in pellet form. The concentration of 5-FU in the supernatant was determined spectrophotometrically at a wavelength of $267 \mathrm{~nm}$ and entrapment efficiency is given by

Total drug - Free drug

Entrapment efficiency $=\frac{\text { Total drug }}{\text { X } 100 \ldots \ldots \ldots \ldots \ldots \text { Equation } 1 .}$.

DQN in the supernatant was quantified by HPLC appended with a fluorescence detector (FP2020, Jasco) and an autosampler (AS-2055 Plus, Jasco) on a $\mathrm{C}_{18}$ reverse phase column (5 $\mu \mathrm{m}$, $250 \mathrm{~mm}$ X $4.6 \mathrm{~mm}$ i.d, Purospher ${ }^{\circledR}$ Star RP-18) as per equation 1. The mobile phase consisted of methanol: water in a ratio of $87: 13 \mathrm{v} / \mathrm{v}$, containing $1.76 \mathrm{mM}$ calcium chloride dihydrate. The analysis was carried out at a flow rate of $0.7 \mathrm{~mL} / \mathrm{min}$ using a $20 \mu \mathrm{L}$ loop.

\subsubsection{Particle size and zeta potential measurements}

Particle size and zeta potential measurements of the formulations were carried out at $25^{\circ} \mathrm{C}$ with the aid of DLS on Malvern Nano-ZS 90 particle size analyser.

Besides, particle size analysis at elevated temperatures in the range of $25{ }^{\circ} \mathrm{C}$ to $70{ }^{\circ} \mathrm{C}$ was also conducted for GMS vesicles with and without surface modification on Horiba particle size analyser (Horiba Zetasizer). The samples were suitably diluted prior to measurements conducted on both particle size analysers. The results have been expressed as a measure of five replicates.

\subsubsection{Morphology: TEM imaging}

GMS vesicles were visualized on a Philips CM200 TEM at an accelerating voltage of $200 \mathrm{keV}$ using phosphotungstic acid as a contrast agent. Briefly, $5 \mu \mathrm{L}$ of the formulation was placed on a carbon coated copper grid (\#400). The latter was then stained, air dried and visualized for morphology at ambient temperature.

2.3.5 Small-angle neutron scattering (SANS) 
SANS measurements were carried out to investigate morphology and bilayer thickness of GMS vesicles and surface modified GMS vesicles. SANS measurements were performed at $30{ }^{\circ} \mathrm{C}$, $45{ }^{\circ} \mathrm{C}$ and $60{ }^{\circ} \mathrm{C}$ on a SANS diffractometer at Guide tube laboratory, Dhruva reactor, Bhabha Atomic Research Centre, Mumbai, India according to the procedure reported by Date et al. ${ }^{16}$. The SANS diffractometer facilitates measurement of neutron scattering intensity as a function of scattering vector, $Q[=(4 \pi / \lambda) \sin (\theta / 2)$, where $\lambda$ is the wavelength of the incident neutrons and $\theta$ is the scattering angle], with the sample placed in a rectangular quartz cell of $2 \mathrm{~mm}$ thickness. The mean wavelength of the monochromatized beam used was $5.2 \AA$ with a spread of $\Delta \lambda / \lambda \sim 10 \%$. The angular distribution of neutrons scattered by the sample was recorded using a $1 \mathrm{~m}$ long one-dimensional He-filled position sensitive detector. The instrument covers a $Q$-range of $0.015-0.35 \AA^{-1}$. The data were corrected for background and empty cell contribution and plotted on an absolute scale using standard procedures. All samples were formulated in $\mathrm{D}_{2} \mathrm{O}$. For the pure GMS vesicles, the GMS excipient was at 100 molar percent (it was a pure system) and for the ligand decorated GMS vesicles, the molar ratio was $97.5 \%$ GMS to $2.5 \%$ hepatotropic ligand.

2.3.6 Differential Scanning Calorimetry (DSC) analysis:

Thermal transitions of GMS vesicular systems were characterized using Mettler Toledo STARe DSC instrument. Pure GMS vesicles were composed of GMS excipient at 100 molar percent (it was a pure system) and a molar ratio of $97.5 \%$ GMS to $2.5 \%$ hepatospecific ligand comprised the ligand decorated GMS vesicles. Each sample was weighed before being placing into the aluminium pan, sealed using crimper and subjected to a heating cycle in the range of $0{ }^{\circ} \mathrm{C}$ to $125{ }^{\circ} \mathrm{C}$ at a heating rate of $10{ }^{\circ} \mathrm{C} / \mathrm{min}$. An empty pan was employed as a reference in the instrument. Peak onset temperature and integral (enthalpy) were evaluated using Mettler Toledo STARe software.

2.4 Stability of GMS vesicles: cholesterol vis-à-vis hepatospecific ligand

Stability of GMS vesicles to leakage of water soluble drug, 5-FU, was investigated in the presence of cholesterol and hepatospecific ligand at $10 \mathrm{~mol} \%$. The vesicles containing cholesterol/ligand were subjected to ultracentrifugation (Beckman Coulter Optima Max XP) at $80,000 \mathrm{rpm}, 4{ }^{\circ} \mathrm{C}$ for $1 \mathrm{~h}$ at ' $0 \mathrm{~h}$ ' and ' $24 \mathrm{~h}$ ' after formulation and the amount of drug released in the supernatant was quantified at $267 \mathrm{~nm}$ by UV spectroscopy. The results have been expressed as a measure of two replicates.

2.5 Molecular modelling studies 
We performed 6 molecular dynamics (MD) simulations. The details of the simulation systems are described in table 1.

\subsubsection{Parametrization of drug molecules}

For drug compounds partial charges were derived in accordance with the Amber forcefield methodology by fitting to the electrostatic potential using the RESP procedure ${ }^{17}$. Firstly, the geometry of the compounds was optimized at density functional theory (DFT) level using the Becke B3LYP exchange-correlation functional and the 6-31G* basis set within the Gaussian 03 program $^{18}$. Subsequently, the molecular electrostatic potential was computed for the optimized molecular structure at the same level of theory. The potential obtained through this method was used to calculate the partial atomic charges according to the RESP procedure implemented in ANTECHAMBER ${ }^{19}$.

\subsubsection{Simulation parameters}

For GMS particle and targeting moiety, S-lipids forcefield parameters were derived from lipid parameters ${ }^{20,21}$. The molecular dynamics simulations were conducted under constant pressure (1 Bar) controlled through a Parrinello-Rahman barostat ${ }^{22}$ using a semi-isotropic pressure scheme. The temperature was controlled using the Nosé-Hoover thermostat ${ }^{23,24}$. The temperatures of the solute and solvent were controlled independently. For water, we used the TIP3 water model ${ }^{25}$. Periodic boundary conditions with the usual minimum image convention were used in all three directions. In order to preserve the covalent bond lengths, the linear constraint solver (LINCS) algorithm ${ }^{26}$ was employed and a 2 fs time step was used in all simulations. The Lennard-Jones Interactions were cut off at $1.4 \mathrm{~nm}$, and for the electrostatic interactions we employed the particle mesh Ewald method ${ }^{27}$. All error bars were calculated using the block method ${ }^{28}$. All simulations were performed using the GroMACS software package, version $4.6 .7^{29}$ and all visualizations were performed using the VMD software package $^{30}$. Analyses were performed using standard analysis tools found in GroMACS as well as previously developed in-house protocols (for solvent accessible surface area analysis) ${ }^{31}$. All MD simulations were performed for $600 \mathrm{~ns}$, of which the first $100 \mathrm{~ns}$ of simulations were treated as equilibration. The analysis was performed on the latter $500 \mathrm{~ns}$ of the trajectory.

\section{RESULTS AND DISCUSSION}


3.1 The potential of GMS molecules to form vesicles: theory and MD simulation

Observing the structue of GMS (see Figure 1), it can clearly be seen to be a single tailed amphiphilic molecule with emulsifying properties that we hypothesize to be capable of forming vesicles; It has an HLB of 3.8. The mechanism of vesicle formation can be explained in congruence with the theory proposed by Kunitake et al, who demonstrated synthetic bilayer membrane formation for one to four tailed amphiphiles. They have suggested that intermolecular interactions in the bilayer morphology of a single tailed amphiphile are governed by the rigid segment of the amphiphile. Intra- and intermolecular interactions, e.g. hydrogen bond formation, have a significant impact on the headgroup cross sectional area available for hydration. The presence of multiple hydrogen bonds reduces the headgroup area available for hydration, leading to vesicle formation vis-à-vis micellization, as demonstrated by Doren $e t a l^{32}$. Apart from inter- and intramolecular H-bonding ${ }^{15}$ demonstrated by Mishra $e t$ $a l$, GMS has a small headgroup area per lipid, even when present as a monolayer. Kawaguchi et al demonstrated the area per GMS molecule to be $0.26 \mathrm{~nm}^{2}$, when present as a liquidcondensed monolayer at the air-water interface ${ }^{33}$.

GMS vesicle formation can also be rationalized based on Israelachvili's expression of critical packing parameter (CPP), $P$, which has been successfully used for predicting the geometry of amphiphiles, and further determines their morphology. The value of the CPP is given by

$P=v / a_{0} l_{c}$ Equation 2.

where, $v$ is the volume of fluid and incompressible hydrocarbon chains, $a_{0}$ is the crosssectional headgroup area per surfactant molecule exposed to the aqueous phase and $l_{c}$ is the critical chain-length. Tanford derived equations for obtaining approximate values of $v$ and $l_{c}$ for a saturated hydrocarbon chain of $n$ carbon atoms, as ${ }^{34}$

$v \cong(27.4+26.9 n) \AA^{3}$ Equation 3.

and

$l_{c} \cong l_{\max } \cong(1.5+1.265 n) \AA$ Equation 4.

In the present work, a value of $P$ of 0.8 was calculated for GMS, using $a_{0}=26 \AA^{2}, v=$ $511.6 \AA^{3}$ and $l_{c}=24.27 \AA$, wherein, $v$ and $l_{c}$ were computed using $n=18$ in equations 3 and 4 respectively. The vesicular structures for amphiphiles are typically defined as being in the 
range $1 / 2<P<1$ and a value of 0.8 lies well within this range, thus substantiating vesicular packing for GMS aggregates in water ${ }^{35}$.

Another factor that governs the shape assumed upon self-assembly is aggregation number, $N$, with smaller aggregation numbers ( 150) suggesting micelle formation, while a larger value (> 1200) indicates a vesicular assembly ${ }^{36,37,38}$. The aggregation number of GMS vesicles can be calculated as

$N=\frac{4 \pi\left[r_{c}^{2}+\left(r_{c}-\frac{2 v}{a_{0}}\right)^{2}\right]}{a_{0}}$ Equation 5.

where $r_{c}$ is the critical radius given by

$r_{c}=\frac{l_{c}}{1-P}$ Equation 6.

The critical radius is the radius of the smallest vesicle possible, and calculated for our system of GMS vesicles to be $12.13 \mathrm{~nm}$, leading to a value of $N=10,036$.

To provide additional evidence that GMS is capable of forming vesicles, we performed an MD simulation of the spontaneous formation of a vesicle from 288 GMS molecules. The GMS molecules were placed within the simulation box randomly, then water molecules were added. Upon simulation under semi-isotropic pressure conditions, we observed that GMS particles self-assemble spontaneously into a membrane bilayer at $80^{\circ} \mathrm{C}$. As shown in Figure 2, GMS molecules interact with each other such that hydrophobic-tail and water contacts are reduced as head group (with oxygen atoms) water contacts are maximized. After $\sim 10$ ns the GMS assembles into structure that resembles a micelle and this then, more slowly, transforms into a stable membrane bilayer, completing this transformation in $\sim 150 \mathrm{~ns}$.

3.2 Surface modification of the GMS vesicle: addition of a targeting moiety

We then investigated surface modification of the GMS vesicle through functionalization of the GMS molecules: a hepatotropic triterpenoid was esterified to a monoglyceride lipid anchor to yield the targeting moiety: a hepatotropic ligand. Negishi et al. have demonstrated the presence of specific triterpenoid receptors on the cell membrane of rat hepatocytes ${ }^{39}$. These receptors, overexpressed in hepatocellular carcinoma ${ }^{40}$, have become an attractive target for drug delivery to hepatocytes. The targeting moiety employed in the present study was thus synthesized with the objective of targeting hepatocytes ${ }^{41}$. It was purified through a preparative TLC technique and its synthesis was confirmed by spectral techniques, e.g. IR, NMR and MS. A molecular 
weight of $810 \mathrm{~g} / \mathrm{mol}$ was obtained using MS; strong IR bands seen at $1735 \mathrm{~cm}^{-1}$ corresponding to $\mathrm{C}=\mathrm{O}$ stretches of both the ester and cyclic ketone at $1647 \mathrm{~cm}^{-1}$ corroborate product formation. The ${ }^{13} \mathrm{C}$-NMR spectrum demonstrated the presence of an ester carbonyl carbon peak at $177 \delta$, while monoglyceride protons ( $\left.\mathrm{R}-\mathrm{CH}_{2}-\mathrm{OCO}\right)$ chemically adjacent to the esterified hydroxy appeared as a singlet at $3.47 \delta$ in ${ }^{1} \mathrm{H}-\mathrm{NMR}$ spectrum, thus demonstrating that synthesis of the targeting moiety has occurred. Also, the targeting moiety was found to be safe and biocompatible in an in vitro HepG2 cytotoxicity study and a 14-day acute toxicity study performed after intravenous administration of the targeting moiety in Swiss albino mice.

To complement our experimental results, we performed MD simulation of GMS vesicles with the aforementioned targeting moiety. The simulation of the GMS membrane bilayer was begun at the elevated temperature of $80{ }^{\circ} \mathrm{C}$ with the targeting moiety placed in the water phase; after $200 \mathrm{~ns}$ of simulation, the temperature was reduced in $20 \mathrm{~ns}$ steps of $10{ }^{\circ} \mathrm{C}$, to finally reach $30{ }^{\circ} \mathrm{C}$. The resulting structure was simulated for $500 \mathrm{~ns}$. The representative snapshot from the 500 ns simulation at $30{ }^{\circ} \mathrm{C}$ is shown in Figure 3, where the GMS tail is shown in magenta and the targeting moiety is shown in orange. The targeting moiety does not enter the membrane bilayer but remains in the membrane headgroup region (Figure 3). Using a Solvent accessible surface area (SASA) analysis we were able to determine that, $\sim 56 \%$ of the targeting moiety is in contact with water, thus accessible to the target receptor, and $\sim 41 \%$ of the targeting moiety remains in contact with the GMS headgroup, whereas only $~ 3 \%$ of it is in contact with GMS tails. This result demonstrates that the GMS membrane vesicle can be functionalized with the targeting moiety, in this case when attached to a monoglyceride tail; computational results provide evidence that this targeting moiety could be effective.

3.3 Size, structure, morphology and amphiphile phase of the GMS vesicles

We studied the particle size of the system at different temperatures with a view to gain insight into the temperature dependent alterations in vesicular membranes. The size of GMS vesicles was found to be $250.0 \pm 16.7 \mathrm{~nm}$ with a PI of 0.4 and zeta potential of $-60 \mathrm{mV}$, when determined at $25^{\circ} \mathrm{C}$ by DLS. However, the particle size increased from $250 \pm 16.7 \mathrm{~nm}$ at $25{ }^{\circ} \mathrm{C}$ to $995.8 \pm$ $172.6 \mathrm{~nm}$ at $40{ }^{\circ} \mathrm{C}$, following which, a precipitous decline to $100 \pm 17 \mathrm{~nm}$ was observed as the temperature was slowly increased up to $70{ }^{\circ} \mathrm{C}$. Ligand modified GMS vesicles also demonstrated similar trends in particle size with increasing temperature, see table 2 . These results indicated two characteristics of GMS vesicles: 1 . There exists a transition region for GMS vesicles, that is, the region between the gel and fluid (liquid crystalline) phases spanning 
a large temperature range; over this temperature change the particle size changes considerably. This is in accordance with the reported existence of such a region for DMPC and DMPG vesicles by Enoki et $a l^{42}$; surface modification of GMS vesicles does not alter its response to particle size in the transition region.

Vesicles are formed owing to the interplay of opposing forces, both attractive and repulsive. They are characterized by the presence of a phase transition temperature, reflecting lipid-lipid interactions in vesicles. At this temperature, vesicles undergo transition from the gel phase to a more fluid liquid crystalline phase, with the vesicles being softer, more deformable and less stressed by membrane curvature in the fluid phase. Vesicular structures, sometimes, display the existence of a transition region, which extends over a broad temperature range, and is marked by significant alterations in particle size, as observed in GMS vesicles. A considerable increase in particle size observed at $40{ }^{\circ} \mathrm{C}$ for GMS vesicles can be explained by the holey vesicle theory, first proposed by Alakoskela $e t a l^{43}$ and further developed by Enoki et al. The theory emphasizes the presence of perforated vesicles in the transition region and simultaneous decrease in spontaneous curvature, resulting in an increased particle size due to fusion ${ }^{42,44}$. This theory is also supported by Bagatolli and Gratton who have demonstrated shape and size hysteresis with temperature variation ${ }^{45}$. Their hypothesis suggests the existence of membrane defects (pores) which allow water movement with increasing temperature corroborating changing vesicle diameter for giant unilamellar vesicles (GUVs). As the temperature is increased beyond the transition range, GMS vesicles plausibly lose water as they become more fluid and shrink to a size of around 100 to $120 \mathrm{~nm}$. Particle size alterations with increasing temperature suggest the existence of a transition region characteristic of vesicular systems, indicating formation of vesicles in the present study.

Morphological investigation of the formulated system and corroboration of its particle size obtained by DLS was additionally carried out through TEM imaging studies. TEM images shown in Figure 4 evinced unilamellar, spherical vesicular structures in the range of 150 to $200 \mathrm{~nm}$, confirming vesicle formation for GMS nanosystems. The particle size obtained by TEM was in congruence with the values obtained by DLS measurements. The background in the TEM images appears to be fuzzy probably due to the drying process used in our TEM studies. For example, Klein et al. have also reported such discontinuity in the background obtained on recording TEM images for gold nanoparticles. They have ascribed such discontinuity in the background to the dried residue of the dispensing solution upon desiccation ${ }^{46}$. 
Further, SANS measurements were carried out on GMS and surface modified GMS vesicles to deduce morphology and changes in the bilayer thickness with temperature using a SANS diffractometer. Data analysis was performed using SASfit software with a unilamellar vesicle model. The absence of lower cut-offs in the data indicates that the radii of the vesicles could be much larger than what can be determined from the present low $Q$ limit (i.e. $2 \pi / Q_{\min }$ ). Therefore, the radius of vesicles was kept fixed at $2 \pi / Q_{\text {min }}, \sim 400 \AA$. The data were analyzed by comparing the scattering from different models to the experimental data. Throughout the data analysis, corrections were made for instrumental smearing, where the calculated scattering profiles were smeared by an appropriate resolution function to compare with the measured data $^{47}$. The fitted parameters in the analysis were optimized using the nonlinear least-square fitting to the model scattering ${ }^{48}$. A slope of (-2) was obtained from the plots of scattering intensity vs scattering angle, $Q$, for conventional and surface modified GMS vesicles, corroborating vesicle formation ${ }^{49,50}$. The absence of Bragg peaks in these plots suggested that only unilammelar vesicles were present. Bilayer thickness measurements for plain and surface modified GMS vesicles are as shown in table 3. Both types of vesicles showed a $20 \AA$ increase in bilayer thickness when the temperature was raised from $30{ }^{\circ} \mathrm{C}$ to $60{ }^{\circ} \mathrm{C}$ as shown in Figure 5. However, a minimal $(\sim 1 \AA)$ increase was noted at $45{ }^{\circ} \mathrm{C}$. This indicated changes in bilayer thickness due to phase transition occur at around $60{ }^{\circ} \mathrm{C}$, compliant with particle size alterations obtained by DLS measurements at higher temperatures. We thus observe the opposite to occur as what has been observed for DMPC ${ }^{51}$ and DPPC vesicles ${ }^{52,53,54}$, where a decrease in bilayer thickness has been observed with increasing temperature as the vesicle transitions from the ordered gel phase to the more disordered liquid crystalline phase, attributed to the comparative effect on acyl chain order and lipid tilt, governing bilayer thickness. Similarly, the influence of the presence of the targeting moiety on the acyl chain order of the GMS bilayer could be responsible for the observed $2 \AA$ difference in the bilayer thickness of the surface modified GMS vesicles and plain GMS vesicles in the gel phase.

To complement the findings from the SANS studies regarding membrane thickness, we next performed MD simulations of a GMS membrane bilayer at $30{ }^{\circ} \mathrm{C}$ and $60{ }^{\circ} \mathrm{C}$. As shown in Figure 6, with increase in temperature from $30^{\circ} \mathrm{C}$ to $60{ }^{\circ} \mathrm{C}$, membrane bilayer thickness increases, however the GMS vesicles remain in the gel state, evident from the lipid ordering observed. At $60{ }^{\circ} \mathrm{C}$, the average GMS tilt angle, defined as the angle between the GMS lipid tail atoms and the membrane normal decreases from $27.3^{\circ}(+/-3.9)$ to $11.4^{\circ}(+/-8.2)$, causing the increase in membrane thickness from $39.6 \AA$ to $49.6 \AA$. This can be caused due to an 
increase in the intermolecular thermal motion, as the transition temperature for GMS is beyond $60{ }^{\circ} \mathrm{C}$ and the membrane remains in the gel state.

The variable-temperature SANS and modelling studies helped define the temperature range over which the phase transition occurs for both plain and surface modified vesicles. Accurate measurement of the transition temperatures, however, were made with the aid of DSC. The occurrence of a phase transition is often indicated by an endothermic transition in a thermoanalytical curve; this was observed to occur at $62.35{ }^{\circ} \mathrm{C}$ and $60.27{ }^{\circ} \mathrm{C}$, as shown in Figure 7, for the conventional and ligand modified GMS vesicles respectively. Mabrey and Sturtevant reported an upper transition temperature of $54.9^{\circ} \mathrm{C}$ for DSPC multilamellar vesicles in water ${ }^{55}$. Phase transition entails chain disordering by overcoming the anisotropic restraints posed by the orientation of hydrocarbon chains in the gel phase of the vesicles. A slightly higher transition temperature observed for GMS vesicles could be attributed to a more rigid headgroup vis-à-vis DSPC, in addition to the presence of stearyl chains, which together impose a higher energy barrier for chain rotation to occur, as explained by Taylor and Morris ${ }^{56}$. The small reduction in the transition temperature that results from the surface modification of the GMS vesicle with the targeting moieties could be due to local disordering in the gel phase of GMS vesicles, similar to that produced by the presence of cholesterol in phospholipid bilayers. This is compliant with the effect of targeting moieties on the chain order in the gel phase, indicated by SANS results in the form of a difference in bilayer thickness. No other noticeable endothermic transitions were evident in the thermoanalytical curve of the GMS vesicles. DSC analysis further corroborated SANS results and the existence of vesicular structures could be construed from the data.

3.4 Drug loading and stability of GMS vesicles: potential as drug nanocarriers

Now that we have characterized the properties of GMS vesicles and shown their potential for decoration with hepatospecific ligands, we now further explore their potential as drug delivery nanocarriers; we explore their capacity for loading both hydrophilic and hydrophobic drugs along with other relevant characteristics including trapped volume and physical stability upon drug loading.

Vesicular structures are essentially bilayers with a characteristic aqueous core, thus allowing for the loading of hydrophilic, in addition to hydrophobic, molecules ${ }^{57}$. The drug, 5-FU, a water-soluble drug, was incorporated into GMS vesicles using the thin film hydration method. Sephadex gel- based separation demonstrated $7 \%$ entrapment efficiency for 5-FU as quantified 
using UV spectroscopy, thus indicating presence of aqueous phase within GMS nanosystems. This is comparable to the 2 to $6 \%$ entrapment efficiency obtained with liposomes of different lipid compositions ${ }^{58}$. Being water-soluble, 5 -FU has been shown to possess no interaction with the bilayer membrane. Besides, owing to a low trapped to external volume ratio, it is incorporated into vesicular systems with a low entrapment efficiency ${ }^{59}$. The trapped volume was calculated to be $1.26 \pm 0.17 \mathrm{~L} / \mathrm{mol}$ (calculated from encapsulation data, with known solute concentration), which is comparable to that obtained for small unilamellar vesicles of phospholipids ${ }^{60,61}$.

A hydrophobic drug, DQN, was separately incorporated into GMS vesicles using the thin film hydration method. Entrapment efficiency of $\sim 70 \%$ was obtained for drug/lipid loading of $0.8 \mathrm{~mol} \%$, when estimated by HPLC equipped with fluorescence detector, thus indicating propensity of GMS vesicles to serve as hydrophobic drug carriers. DQN probably interacts with the bilayer membrane of GMS vesicles, resulting in $70 \%$ entrapment efficiency.

Physical stability of vesicles is often compromised due to drug leakage, thus warranting the formulation of more stable vesicular systems ${ }^{62}$. In view of this, the stability of GMS vesicles to 5-FU leakage was determined in the presence of both cholesterol and the hepatospecific ligand. Cholesterol is often included in the formulation of drug delivery liposomes to optimize the rigidity and permeability of the membrane. It was found that cholesterol containing GMS vesicles entrapped less 5-FU than both ligand modified and plain GMS vesicles. The drug was, however, retained, even after 24 hours, to the extent of $93 \%$ in cholesterol containing GMS vesicles and $\sim 90 \%$ in plain GMS vesicles. This is in stark contrast to the vesicles containing the ligand where 5-FU leakage from the vesicles was $50 \%$ in 24 hours, as shown in Figure 8 and table 4 . Solute leakage from vesicular systems is governed by the membrane permeability of the solute and the lipid composition ${ }^{63}$. It can be construed that the permeability of 5-FU through GMS vesicles would bear an insignificant effect on its leakage through the bilayer membrane, owing to high water solubility and low lipid permeability of 5-FU ${ }^{64}$. Lipid composition is, however, seen to markedly influence 5-FU leakage through GMS vesicles; GMS, a $\mathrm{C}_{18}$-based lipid, is analogous to a saturated $\mathrm{C}_{18}$ phospholipid, that is less permeable to entrapped solutes; as a result, they are more stable to solute leakage ${ }^{62}$. The probable mechanism through which the presence of cholesterol decreased leakage from the vesicle was increasing the rigidity of the membrane. The increased leakage when the vesicle was decorated with the hepatospecific ligands probably results from the introduction of disorder in the GMS bilayer, 
increasing the fluidity of the vesicle membrane, leading to increased contact of 5-FU with the external aqueous phase. The ligand/GMS interaction in the bilayer is evident in the molecular dynamics simulations, shown in Figure 3.

The interactions of hydrophobic drug, DQN and hydrophilic drug 5-FU with the GMS membrane bilayer were studied by molecular modelling and analyzed as shown in Figure 9. We see, as expected, the DQN drug molecules enter the GMS membrane bilayer to interact with GMS tails and do not present themselves at the GMS-water interface, evident from the mass density profile and shown in a representative snapshot (see Figure 9). In contrast, the hydrophilic drug 5-FU does not enter the GMS membrane; it interacts with the GMS membrane headgroup or remains in the bulk water (also shown in Figure 9). These results show that, when GMS vesicles are used as a drug carrier, they, like phospholipid vesicles, have the capacity to load hydrophobic molecules, which will enter into the membrane bilayer, in addition to hydrophilic drug molecules, that enter the aqueous core of the vesicles.

\section{CONCLUSION}

Plain and surface modified GMS vesicles were investigated for vesicular properties and applications as drug delivery vehicles. Self-assembly of the single-tailed GMS molecules was confirmed experimentally through SANS and demonstrated through molecular dynamics simulation studies. Both our experimental and computational results provide evidence that vesicles formed from these lipids are amenable to surface modification. Visualization by TEM imaging unveiled the presence of spherical vesicular morphology. Particle size measurements revealed marked changes in the transition region, supported by SANS and in silico studies. Phase transition temperature for the formulated GMS vesicles was determined though DSC. Further, GMS vesicles displayed the presence of an aqueous core when loaded with a hydrophilic drug and demonstrated bilayer-drug interaction for a hydrophobic drug, adjudged in a simulation study and determined experimentally by ultracentrifugation. While surface modification of GMS vesicles augmented encapsulation of a water-soluble drug, addition of cholesterol reduced its leakage from the vesicles. GMS vesicles, capable of surface functionalization and loading of both hydrophobic and hydrophilic drugs, augur exciting potential as a phospholipid surrogate and open avenues in the realm of membrane mimetic chemistry and formulation of one-tailed amphiphiles into drug nanocarriers with active targeting. 


\section{ACKNOWLEDGMENTS}

Computational resources have been provided by the CSC-IT center for science Ltd. (CSC). We wish to thank the Finnish Cultural foundation (A. M.) and the Magnus Ehrenrooth foundation (A. B. and A. M.) for funding. A. B. wishes to thank the Academy of Finland, mobility grant \#298827. The authors would like to acknowledge Council of Scientific and Industrial Research and Amrut Mody Research Fund for financially supporting the project. Megha Marwah is thankful to Council of Scientific and Industrial Research and Amrut Mody Research Fund for providing fellowship. The authors would like to thank SAIF, IIT Bombay for carrying out TEM imaging of the formulation samples.

Tables and Figures:

Table 1: Details of the simulation systems used in molecular dynamics studies.

\begin{tabular}{|c|c|c|c|c|c|c|}
\hline System & Aim & & & plecules & & \\
\hline & & GMS & Decoquinate & 5-Fluorouracil & $\begin{array}{c}\text { Targeting } \\
\text { moiety }\end{array}$ & Water \\
\hline 1 & $\begin{array}{l}\text { Self-assembly of } \\
\text { GMS membrane }\end{array}$ & 288 & 0 & 0 & 0 & 11557 \\
\hline 2 & $\begin{array}{c}\text { Drug with GMS } \\
\text { membrane }\end{array}$ & 288 & 12 & 0 & 0 & 11557 \\
\hline 3 & $\begin{array}{c}\text { Drug with GMS } \\
\text { membrane }\end{array}$ & 288 & 0 & 12 & 0 & 11557 \\
\hline 4 & $\begin{array}{c}\text { Targeting moiety } \\
\text { with GMS membrane }\end{array}$ & 288 & 0 & 0 & 28 & 11557 \\
\hline
\end{tabular}

Table 2: Changes in particle size of GMS vesicles and ligand decorated GMS vesicles with temperature $(\mathrm{n}=5)$

\begin{tabular}{|c|c|c|}
\hline $\begin{array}{c}\text { Temperature } \\
\text { in }{ }^{\circ} \mathrm{C}\end{array}$ & $\begin{array}{c}\text { Z-average size of ligand } \\
\text { decorated GMS vesicles in } \mathrm{nm}\end{array}$ & $\begin{array}{c}\text { Z-average size of GMS } \\
\text { vesicles in } \mathrm{nm}\end{array}$ \\
\hline 15 & $617.40 \pm 32.17$ & $652.64 \pm 22.65$ \\
\hline 25 & $205.64 \pm 17.56$ & $250.88 \pm 16.67$ \\
\hline 40 & $1348.16 \pm 658$ & $995.8 \pm 172.59$ \\
\hline 50 & $96 \pm 14.49$ & $120.1 \pm 13.01$ \\
\hline 55 & $107.30 \pm 8.77$ & $114.64 \pm 4.66$ \\
\hline 57 & $103.03 \pm 12.15$ & $102.22 \pm 5.9$ \\
\hline
\end{tabular}




\begin{tabular}{|c|c|c|}
\hline 60 & $109.45 \pm 0.07$ & $97.12 \pm 5.26$ \\
\hline 62 & $104.02 \pm 6.95$ & $106.86 \pm 6.12$ \\
\hline 65 & $110.82 \pm 12.61$ & $97.92 \pm 13.8$ \\
\hline 67 & $120.83 \pm 2.38$ & $101.56 \pm 17.81$ \\
\hline
\end{tabular}

Table 3: Bilayer thickness variation of GMS vesicles and ligand decorated GMS vesicles with temperature

\begin{tabular}{|c|c|c|}
\hline Formulation & Temperature $\left({ }^{\circ} \mathbf{C}\right)$ & Bilayer thickness $\boldsymbol{t}(\mathbf{\AA})$ \\
\hline \multirow{2}{*}{ GMS vesicles } & 30 & $41.3 \pm 0.5$ \\
\cline { 2 - 3 } & 45 & $42.0 \pm 0.5$ \\
\cline { 2 - 3 } & 60 & $63.7 \pm 0.7$ \\
\hline \multirow{2}{*}{$\begin{array}{c}\text { Ligand decorated } \\
\text { GMS vesicles }\end{array}$} & 30 & $39.2 \pm 0.5$ \\
\cline { 2 - 3 } & 45 & $41.0 \pm 0.5$ \\
\cline { 2 - 3 } & 60 & $63.6 \pm 0.7$ \\
\hline
\end{tabular}

*Vesicle radius $>400 \AA$

Table 4: Entrapment efficiency of different GMS vesicles: comparison $(\mathrm{n}=2)$

\begin{tabular}{|l|l|}
\hline Formulation & Percent entrapment efficiency (\%) \\
\hline GMS vesicles & $4.18 \pm 0.077$ \\
\hline GMS vesicles with cholesterol & $2.70 \pm 0.075$ \\
\hline GMS vesicles with ligand & $10.30 \pm 0.012$ \\
\hline
\end{tabular}

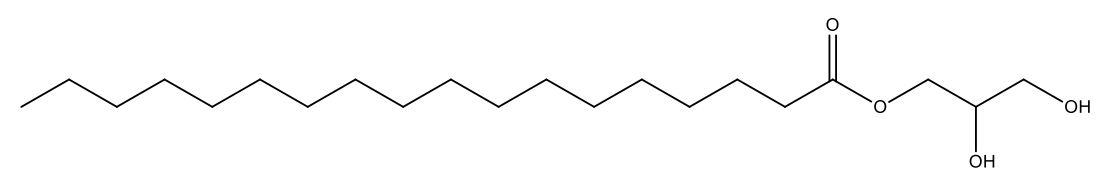

Figure 1: Structure of GMS 


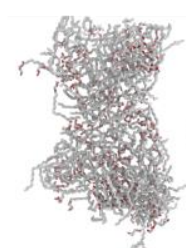

o ns

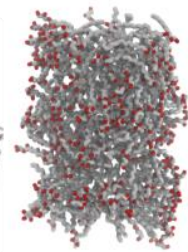

$5 \mathrm{~ns}$

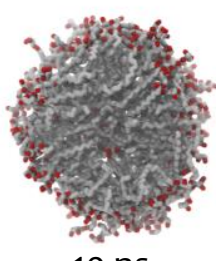

$10 \mathrm{~ns}$

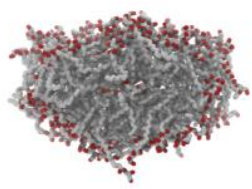

$70 \mathrm{~ns}$

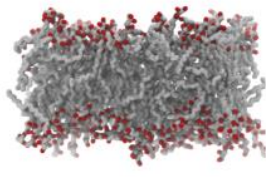

$150 \mathrm{~ns}$

Figure 2: Self-assembly of GMS particles into membrane bilayer at $80^{\circ} \mathrm{C}$ (hydrogen atoms and water molecules are not shown for clarity).
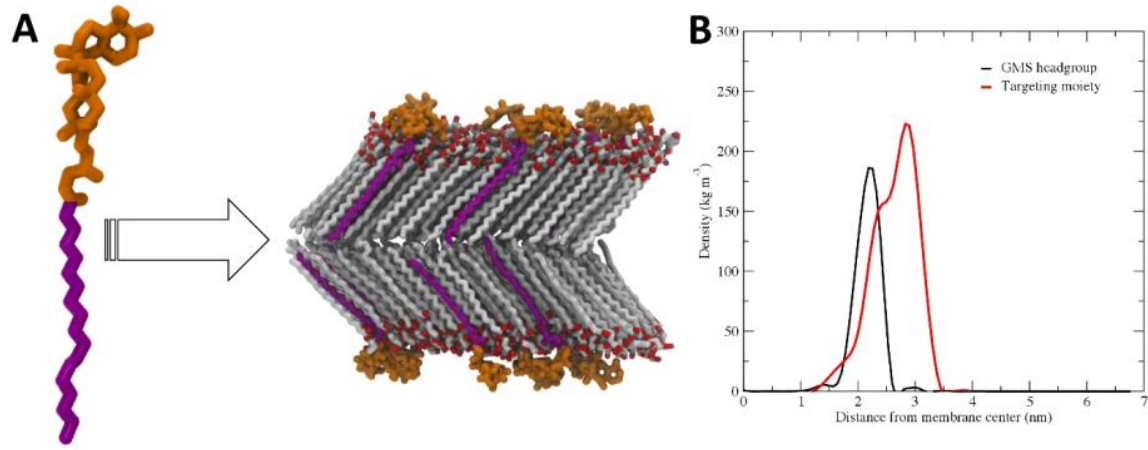

Figure 3: Representative snapshot of GMS membrane bilayer with targeting moiety, (A) GMS tail is shown in magenta and targeting moiety is shown in orange (hydrogen atoms and water molecules are not shown for clarity). (B) Mass density profile for GMS membrane bilayer with the targeting moiety. 


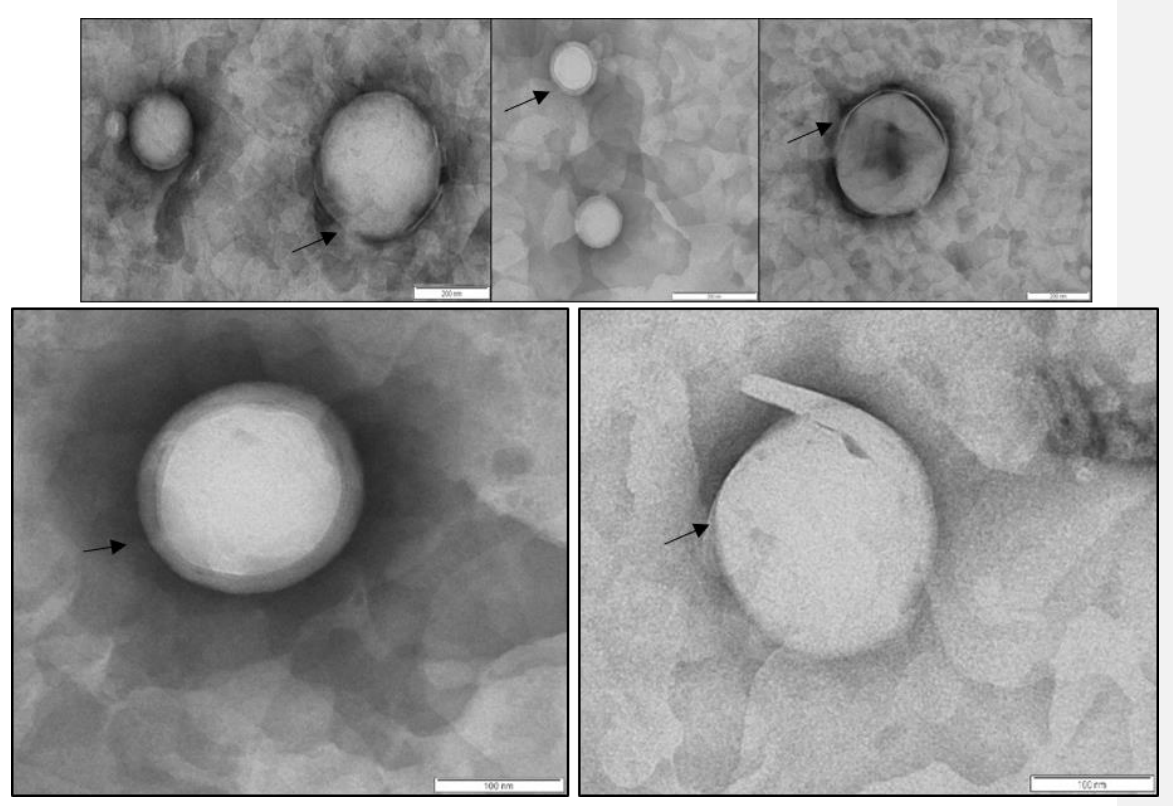

Figure 4: TEM images of plain GMS vesicles (arrow showing bilayer in GMS vesicles).

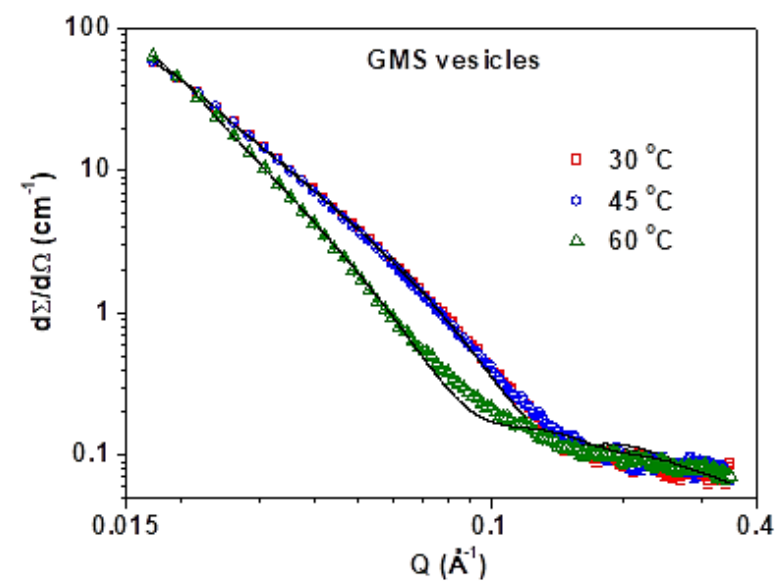




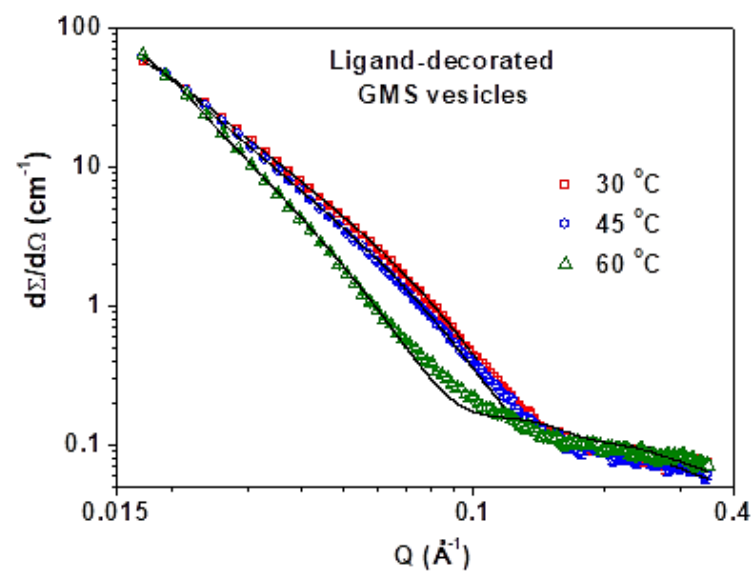

Figure 5: SANS plots of plain and modified GMS vesicles at $30{ }^{\circ} \mathrm{C}, 45^{\circ} \mathrm{C}$ and $60{ }^{\circ} \mathrm{C}$

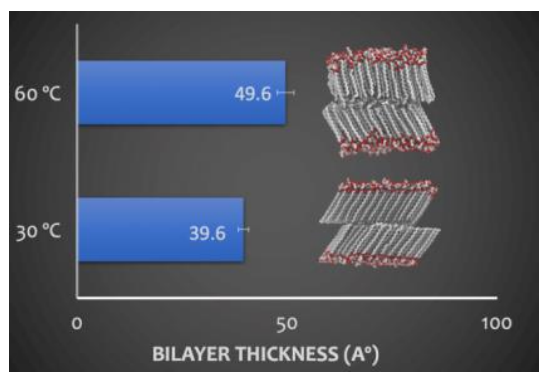

Figure 6: Effect of temperature on GMS membrane bilayer (hydrogen atoms and water molecules are not shown for clarity). 
(a)

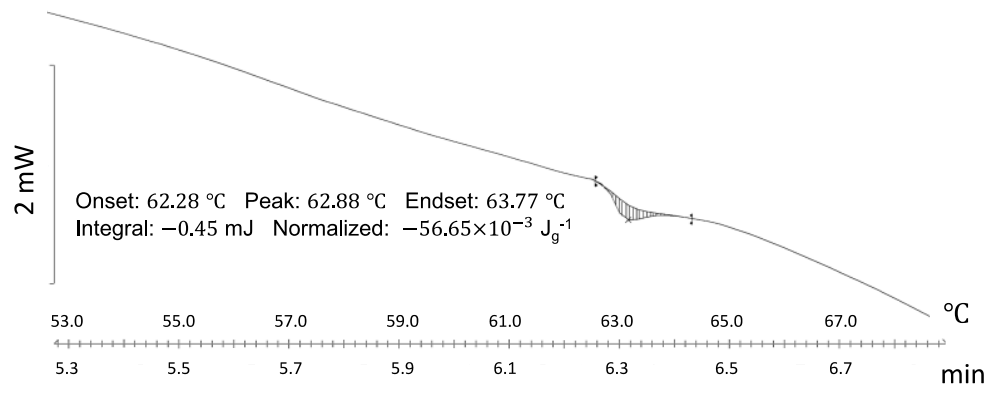

(b)

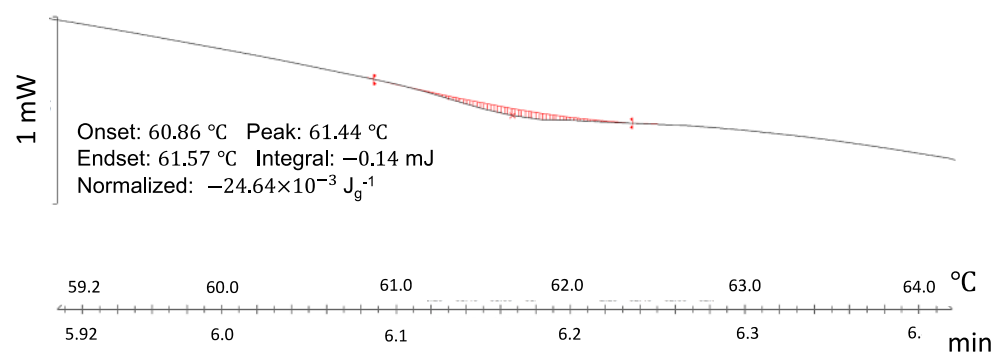

Figure 7: DSC of plain (a) and surface modified (b) GMS vesicles.

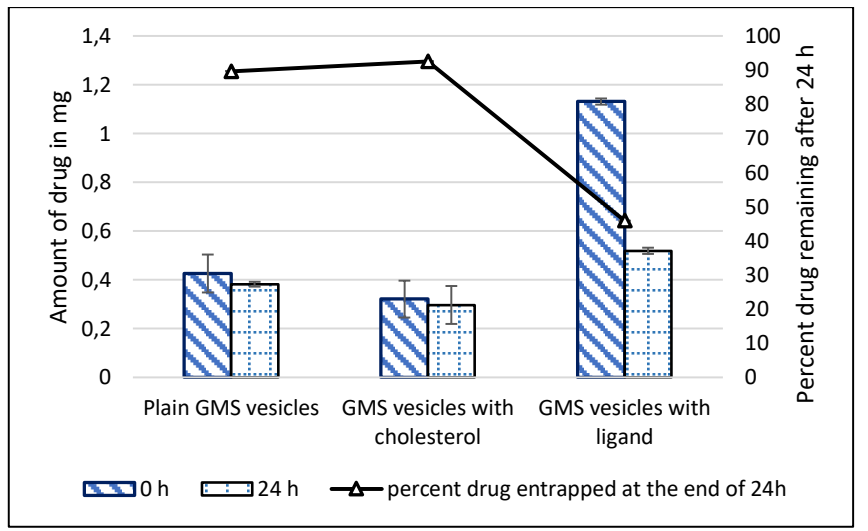

Figure 8: Physical stability of different GMS vesicles over 24 hours $(n=2)$ 


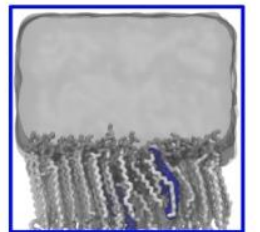

Decoquinate in GMS membrane
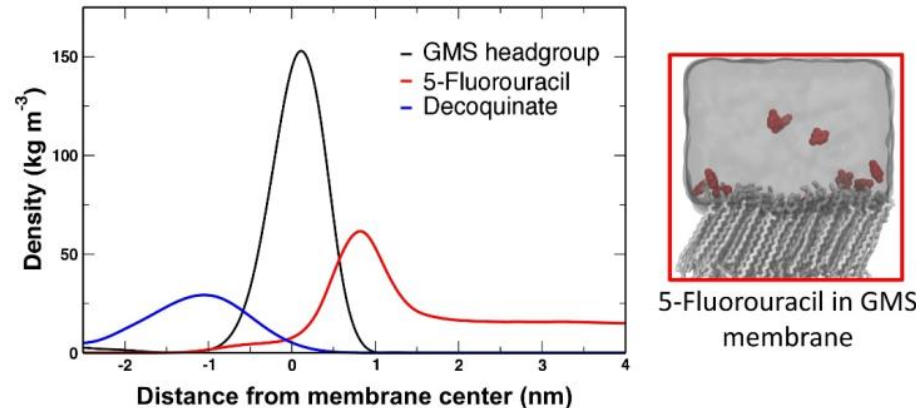

5-Fluorouracil in GMS membrane

Figure 9: Mass density profile of hydrophobic drug, Decoquinate and hydrophilic drug 5-Fluorouracil with GMS membrane headgroup (hydrogen atoms and water molecules are not shown for clarity).

\section{REFERENCES:}

1. Barenholz, Y. C., Doxil ${ }^{\circledR}$-the first FDA-approved nano-drug: lessons learned. J. Controlled Release 2012, 160 (2), 117-134

2. Bunker, A.; Magarkar, A.; Viitala, T., Rational design of liposomal drug delivery systems, a review: combined experimental and computational studies of lipid membranes, liposomes and their PEGylation. Biochim. Biophys. Acta, Biomembr. 2016, 1858 (10), 2334-2352.

3. Kunitake, T., Synthetic Bilayer Membranes: Molecular Design, Self-Organization, and Application. Angew. Chem., Int. Ed. 1992, 31 (6), 709-726.

4. Kunitake, T.; Okahata, Y., Formation of the stable bilayer assemblies in dilute aqueous solution from ammonium amphiphiles with the diphenylazomethine segment. J. Am. Chem. Soc. 1980, 102 (2), 549-553.

5. Kaler, E. W.; Murthy, A. K.; Rodriguez, B. E.; Zasadzinski, J., Spontaneous vesicle formation in aqueous mixtures of single-tailed surfactants. Science 1989, 245 (4924), 1371-1374;

6. Dhawan, V. V.; Nagarsenker, M. S., Catanionic systems in nanotherapeutics-Biophysical aspects and novel trends in drug delivery applications. J. Controlled Release 2017, 266, 331-345

7. Engberts, J. B.; Hoekstra, D., Vesicle-forming synthetic amphiphiles. Biochim. Biophys. Acta, Biomembr. 1995, 1241 (3), 323-340.

8. Sagalowicz, L.; Leser, M.; Watzke, H.; Michel, M., Monoglyceride self-assembly structures as delivery vehicles. Trends Food Sci. Technol. 2006, 17 (5), 204-214.

9. Udaya, S.; Josephine, R.; Kiran, B., Self nano emulsifying drug delivery systems for oral delivery of hydrophobic drugs. Biomed. Pharmcol. J. 2013, 6, 355-362.

10. Kumar, R.; Yasir, M.; Saraf, S. A.; Gaur, P. K.; Kumar, Y.; Singh, A. P., Glyceryl monostearate based nanoparticles of mefenamic acid: fabrication and in vitro characterization. Drug Invent. Today 2013, 5 (3), 246-250.

11. Shenoy, V. S.; Rajyaguru, T. H.; Gude, R. P.; Murthy, R. S. R., Studies on paclitaxel-loaded glyceryl monostearate nanoparticles. Journal. Microencapsulation 2009, 26 (6), 471-478. 
12. Gardouh, A. R.; Gad, S.; Ghonaim, H. M.; Ghorab, M. M., Design and Characterization of Glyceryl Monostearate Solid Lipid Nanoparticles Prepared by High Shear Homogenization. Br. J. Pharm. Res. 2013, 3 (3), 326-346.

13. Pawar, A. Y.; Erande, K. B.; Sonawane, D. D.; Asawale, V. R.; Harak, Y. S.; Derle, D. V., Compressed glyceryl monostearate based biodegradable implant of Gentamicin using melt granulation technique: In vitro evaluation \& biocompatibility in animals. Drug Invent. Today 2013, 5 (1), 39-49.

14. Patil, R. R.; Gaikwad, R. V.; Samad, A.; Devarajan, P. V., Role of lipids in enhancing splenic uptake of polymer-lipid (LIPOMER) nanoparticles. J. Biomed. Nanotechnol. 2008, 4 (3), 359-366. 15. Mishra, P.; Gupta, G. K.; Jain, V., Stearic acid and glyceryl monostearate based selfassembled vesicles: preparation and in vitro evaluation. J. Dispersion Sci. Technol. 2009, 30 (10), 1449-1457.

16. Date, A. A.; Nagarsenker, M. S.; Patere, S.; Dhawan, V.; Gude, R.; Hassan, P.; Aswal, V.; Steiniger, F.; Thamm, J.; Fahr, A., Lecithin-based novel cationic nanocarriers (Leciplex) II: improving therapeutic efficacy of quercetin on oral administration. Mol. Pharmaceutics 2011, 8 (3), 716-726.

17. Bayly, C. I.; Cieplak, P.; Cornell, W.; Kollman, P. A., A well-behaved electrostatic potential based method using charge restraints for deriving atomic charges: the RESP model. J. Phys. Chem. 1993, 97 (40), 10269-10280.

18. Frisch, M.; Trucks, G.; Schlegel, H. B.; Scuseria, G.; Robb, M.; Cheeseman, J.; Montgomery Jr, J.; Vreven, T.; Kudin, K.; Burant, J., Gaussian 03, revision C. 02; Gaussian, Inc. Wallingford, CT 2004,

26.

19. Wang, J.; Wang, W.; Kollman, P. A.; Case, D. A., Automatic atom type and bond type perception in molecular mechanical calculations. J. Mol. Graphics Modell. 2006, 25 (2), 247-260.

20. Jämbeck, J. P.; Lyubartsev, A. P., An extension and further validation of an all-atomistic force field for biological membranes. J. Chem. Theory Comput. 2012, 8 (8), 2938-2948;

21. Jämbeck, J. P.; Lyubartsev, A. P., Derivation and systematic validation of a refined all-atom force field for phosphatidylcholine lipids. J Phys. Chem. B 2012, 116 (10), 3164-3179.

22. Parrinello, M.; Rahman, A., Polymorphic transitions in single crystals: A new molecular dynamics method. J. Appl. Phys. 1981, 52 (12), 7182-7190.

23. Nosé, S., A unified formulation of the constant temperature molecular dynamics methods. J. Chem. Phys. 1984, 81 (1), 511-519

24. Hoover, W. G., Canonical dynamics: equilibrium phase-space distributions. Phys. Rev. A 1985, 31 (3), 1695.

25. Jorgensen, W. L., Chandrasekhar, J., Madura, J. D., Impey, R. W., \& Klein, M. L. Comparison of simple potential functions for simulating liquid water. J. Chem. Phys. 1983, 79 (2), 926-935.

26. Hess, B.; Bekker, H.; Berendsen, H. J.; Fraaije, J. G., LINCS: a linear constraint solver for molecular simulations. J. Comput. Chem. 1997, 18 (12), 1463-1472.

27. Essmann, U.; Perera, L.; Berkowitz, M. L.; Darden, T.; Lee, H.; Pedersen, L. G., A smooth particle mesh Ewald method. J. Chem. Phys. 1995, 103 (19), 8577-8593.

28. Hess, B., Determining the shear viscosity of model liquids from molecular dynamics simulations. J. Chem. Phys. 2002, 116 (1), 209-217.

29. Pronk, S.; Páll, S.; Schulz, R.; Larsson, P.; Bjelkmar, P.; Apostolov, R.; Shirts, M. R.; Smith, J. C.; Kasson, P. M.; Van Der Spoel, D., GROMACS 4.5: a high-throughput and highly parallel open source molecular simulation toolkit. Bioinformatics 2013, 29 (7), 845-854.

30. Humphrey, W.; Dalke, A.; Schulten, K., VMD: visual molecular dynamics. J. Mol. Graphics 1996, 14 (1), 33-38.

31. Lehtinen, J.; Magarkar, A.; Stepniewski, M.; Hakola, S.; Bergman, M.; Róg, T.; Yliperttula, M Urtti, A.; Bunker, A., Analysis of cause of failure of new targeting peptide in PEGylated liposome: molecular modeling as rational design tool for nanomedicine. Eur. J. Pharm. Sci. 2012, 46 (3), $121-$ 130 
32. Doren, H. A. v.; Galema, S. A.; Engberts, J. B., Unexpected Formation of Vesicular Aggregates in Aqueous Solutions of n-Octyl 1-Thio-. alpha.-D-talopyranoside. Marked Effects of Intramolecular Hydrogen Bonding. Langmuir 1995, 11 (2), 687-688.

33. Kawaguchi, M.; Yamamoto, M.; Nakamura, T.; Yamashita, M.; Kato, T.; Kato, T., Surface Properties of Mono-, Di-, and Triglycerol Monostearate Monolayers Spread at the Air- Water Interface. Langmuir 2001, 17 (15), 4677-4680.

34. Tanford, C., Micelle shape and size. J. Phys. Chem. 1972, 76 (21), 3020-3024.

35. Israelachvili, J., Soft and biological structures. Intermolecular and surface forces 2011, 3, 535-

569.

36. Nagarajan, R.; Ruckenstein, E., Aggregation of amphiphiles as micelles or vesicles in aqueous media. J. Colloid Interface Sci. 1979, 71 (3), 580-604.

37. Maulucci, G.; De Spirito, M.; Arcovito, G.; Boffi, F.; Castellano, A. C.; Briganti, G., Particle size distribution in DMPC vesicles solutions undergoing different sonication times. Biophysical journal 2005, 88 (5), 3545-3550;

38. Ghosh, P., Colloid and interface science. PHI Learning Pvt. Ltd.: 2009

39. Negishi, M.; Irie, A.; Nagata, N.; Ichikawa, A., Specific binding of glycyrrhetinic acid to the rat liver membrane. Biochim. Biophys. Acta, Biomembr. 1991, 1066 (1), 77-82.

40. Il'Icheva, T.; Proniaeva, T.; Smetannikov, A.; Pokrovskiĭ, A., Content of progesterone, glucocorticoid and glycyrrhizic acid receptors in normal and tumoral human breast tissue. Vopr. Onkol. 1998, 44 (4), 390-394.

41. Marwah, M. S. Novel Systems for Targeted Drug Delivery to Liver: Design, Formulation and Evaluation. Ph.D dissertation, University of Mumbai, India, 2018.

42. Enoki, T. A.; Henriques, V. B.; Lamy, M. T., Light scattering on the structural characterization of DMPG vesicles along the bilayer anomalous phase transition. Chem. Phys. Llipids 2012, 165 (8), 826-837.

43. Alakoskela, J.-M. I.; Kinnunen, P. K., Thermal phase behavior of DMPG: the exclusion of continuous network and dense aggregates. Langmuir 2007, 23 (8), 4203-4213.

44. Majhi, P. R.; Blume, A., Temperature-induced micelle-vesicle transitions in dmpc- sds and dmpc- dtab mixtures studied by calorimetry and dynamic light scattering. J. Phys. Chem. B 2002, 106 (41), 10753-10763.

45. Bagatolli, L.; Gratton, E., Two-photon fluorescence microscopy observation of shape changes at the phase transition in phospholipid giant unilamellar vesicles. Biophysical Journal 1999, 77 (4), 2090-2101.

46. Klein, K. L., Anderson, I. M., De Jonge, N. Transmission electron microscopy with a liquid flow cell. J. Microsc. 2011, 242 (2), 117-123.

47. Pedersen, J. S.; Riekel, C., Resolution function and flux at the sample for small-angle X-ray scattering calculated in position-angle-wavelength space. J. Appl. Crystallogr. 1991, 24 (5), 893-909. 48. Bevington, P. R., Data Reduction and Error Analysis for the Physical Sciences McGraw Hill Book Co. New York 1969

49. Aswal, V.; Goyal, P., Small-angle neutron scattering from micellar solutions. Pramana 2004, $63(1), 65-72$

50. Yue, B.; Huang, C.-Y.; Nieh, M.-P.; Glinka, C. J.; Katsaras, J., Highly stable phospholipid unilamellar vesicles from spontaneous vesiculation: a DLS and SANS study. J. Phys. Chem. B 2005 109 (1), 609-616

51. Pencer, J.; Nieh, M.-P.; Harroun, T. A.; Krueger, S.; Adams, C.; Katsaras, J., Bilayer thickness and thermal response of dimyristoylphosphatidylcholine unilamellar vesicles containing cholesterol, ergosterol and lanosterol: a small-angle neutron scattering study. Biochim. Biophys. Acta, Biomembr. 2005, 1720 (1), 84-91.

52. Leekumjorn, S.; Sum, A. K., Molecular studies of the gel to liquid-crystalline phase transition for fully hydrated DPPC and DPPE bilayers. Biochim. Biophys. Acta, Biomembr. 2007, 1768 (2), 354365. 
53. Leonenko, Z.; Finot, E.; Ma, H.; Dahms, T.; Cramb, D., Investigation of temperature-induced phase transitions in DOPC and DPPC phospholipid bilayers using temperature-controlled scanning force microscopy. Biophys. J.I 2004, 86 (6), 3783-3793.

54. Mason, P.; Gaulin, B.; Epand, R.; Wignall, G.; Lin, J., Small angle neutron scattering and calorimetric studies of large unilamellar vesicles of the phospholipid dipalmitoylphosphatidylcholine. Phys. Rev. E 1999, 59 (3), 3361.

55. Mabrey, S.; Sturtevant, J. M., Investigation of phase transitions of lipids and lipid mixtures by sensitivity differential scanning calorimetry. Proc. Natl. Acad. Sci. 1976, 73 (11), 3862-3866.

56. Taylor, K. M.; Morris, R. M., Thermal analysis of phase transition behaviour in liposomes. Thermochim. Acta 1995, 248, 289-301.

57. Nagarsenker, M. S.; Marwah, M. S., Liposomes: Concept and Therapeutic Applications. Novel Approaches for Drug Delivery 2016, 52.

58. Ozer, A.; Talsma, H., Preparation and stability of liposomes containing 5-fluorouracil. Int. J. Pharm. 1989, 55 (2-3), 185-191.

59. Kaiser, N.; Kimpfler, A.; Massing, U.; Burger, A.; Fiebig, H.; Brandl, M.; Schubert, R., 5Fluorouracil in vesicular phospholipid gels for anticancer treatment: entrapment and release properties. Int. J. Pharm. 2003, 256 (1-2), 123-131.

60. Haran, G.; Cohen, R.; Bar, L. K.; Barenholz, Y., Transmembrane ammonium sulfate gradients in liposomes produce efficient and stable entrapment of amphipathic weak bases. Biochim. Biophys. Acta, Biomembr. 1993, 1151 (2), 201-215.

61. Yoss, N. L.; Popescu, O.; Pop, V. I.; Porutiu, D.; Kummerow, F. A.; Benga, G., Comparison of liposome entrapment parameters by optical and atomic absorption spectrophotometry. Biosci. Rep. 1985, 5 (1), 1-5.

62. Nounou, M. M.; El-Khordagui, L.; Khalafallah, N.; Khalil, S.; RASHEED, K., Influence of different sugar cryoprotectants on the stability and physico-chemical characteristics of freeze-dried 5-fluorouracil plurilamellar vesicles. 2005.

63. Sabliov, C.; Chen, H.; Yada, R., Nanotechnology and functional foods: effective delivery of bioactive ingredients. John Wiley \& Sons: 2015.

64. Fresta, M.; Villari, A.; Puglisi, G.; Cavallaro, G., 5-Fluorouracil: various kinds of loaded liposomes: encapsulation efficiency, storage stability and fusogenic properties. Int. J. Pharm. 1993, $99(2-3), 145-156$. 